\title{
REDUÇÃO DA ÁREA FOLIAR E O RENDIMENTO DO PEPINO JAPONÊS
}

\author{
Edson Shigueaki Nomura; Antonio Ismael Inácio Cardoso* \\ Depto. de Produção Vegetal - FCA/UNESP, C.P. 237 - CEP: 18603-970 - Botucatu, SP. \\ ${ }^{*}$ Autor correspondente <ismaeldh@fca.unesp.br>
}

RESUMO: Para verificar o efeito da redução da área foliar sobre a produção e qualidade dos frutos de pepino japonês (híbrido Hokuho $n^{0} 2$, enxertado sobre abóbora ‘Excite lkky') cultivado em ambiente protegido, foram avaliados seis tratamentos com cinco repetições e cinco plantas por parcela, no delineamento experimental em blocos ao acaso. O tratamento 1 foi constituído por plantas com crescimento livre; no tratamento 2 (padrão) as plantas foram conduzidas com desbrotas nos ramos laterais; no tratamento 3 as plantas foram conduzidas semelhante ao padrão, mas com eliminação de frutos tortos ainda jovens $(<5 \mathrm{~cm})$; os tratamentos 4,5 e 6 foram conduzidos semelhante ao padrão, mas com retirada de $25 \%, 50 \%$ e $75 \%$ das folhas da planta, respectivamente. Não se observou diferenças significativas para altura de plantas, mas os tratamentos 5 e 6 apresentaram maior número de entrenós, embora menos vigorosos, e menor emissão de ramificações laterais. O tratamento 1, apesar de ter uma maior produção total de frutos, apresentou uma grande quantidade de frutos tortos e maior dificuldade para a colheita. No tratamento 3 observou-se uma maior produção de frutos comerciais, já que eram retirados todos os frutos tortos ainda jovens, o que era compensado pela produção de frutos de maior qualidade e favorecendo a emissão de novas brotações laterais. Houve uma redução na produção e qualidade de frutos, proporcional ao nível de desfolha.

Palavras-chave: Cucumis sativus, desfolha

\section{LEAF AREA REDUCTION AND THE YIELD OF THE JAPANESE CUCUMBER}

\begin{abstract}
In order to verify the defoliation effect on yield and quality of japanese cucumber ('Hokuho' grafted over 'Excite lkky' squash) under protected cultivation, five replicates of five plants per plot were used to evaluate six treatments in a randomized block design. Treatment 1 consisted of free growth plants; treatment 2 (standard) of disprouted plants; treatment 3 , young curved fruits $(<5 \mathrm{~cm})$ removed; treatments 4,5 and 6 , plants removing $25 \%, 50 \%$ and $75 \%$ of their leaves, respectively. There were no differences in plant height, but plants of treatments 5 and 6 had greater number of nodes per plant, although they were smaller and less vigorous, presenting a smaller number of sproutings. Despite having plants with higher total yield, in treatment 1, most of them were curved and the harvest was more difficult. Plants of treatment 3 had a greater commercial yield, because all the young curved fruits were removed and the plants compensated their yield, producing other fruits with better quality and plants had better sprouting. The higher the defoliation level the greater was the yield reduction.

Key words: Cucumis sativus, defoliation
\end{abstract}

\section{INTRODUÇÃO}

O pepino (Cucumis sativus L.) tem crescido de importância na comercialização de hortaliças, sendo muito apreciado e consumido em todo o Brasil. Foram comercializadas 34.508 t no CEAGESP em 1998 (FNP Consultoria \& Comércio, 2000).

O pepino é uma espécie não adaptada ao cultivo sob baixas temperaturas, sendo o desenvolvimento da planta favorecido por temperaturas superiores à $20^{\circ} \mathrm{C}$ (Lower \& Edwards, 1986). Este foi um dos motivos pelos quais os produtores passaram a cultivar pepino em ambiente protegido a partir da década de 80 , sendo que se encontra, atualmente, entre as principais hortaliças, ocupando o segundo lugar, após o tomate (Silva et al., 1995).

Aumentos de produtividade sob este sistema de cultivo, em relação ao cultivo em campo aberto, tem sido relatados no Brasil, variando de $0,3 \%$ a $46,3 \%$ (Reis et al., 1991), $48,5 \%$ (Reis et al., 1992) e de $55,0 \%$ a $79,6 \%$ (Oliveira et al., 1995)

$\mathrm{Na}$ produção de pepino japonês, frutos sem deformidades e retos são os mais valorizados. O entortamento dos frutos pode ser causado por diversos fatores tais como má polinização (Denna, 1973), o contato dos frutos sobre uma superfície que impeça seu crescimento normal, dentre outros.

Kanahama \& Saito (1985a) observaram que a quantidade de frutos tortos aumentava, assim como reduzia o crescimento dos frutos, quando havia insuficiência no suprimento de fotossintetizados devido à redução de área foliar e ao sombreamento.

O entortamento ocorre após uma temporária supressão do crescimento do ovário. O máximo ângulo da curvatura dos frutos ocorria 5 - 6 dias após o florescimento, quando os frutos estavam com $10-15 \mathrm{~cm}$ 
de comprimento ou um pouco antes da colheita (Kanahama \& Saito, 1985b).

Kanahama \& Saito (1988) sugeriram que o entortamento de frutos de pepino é resultado da competição entre os lóculos por fotossintetizados, sendo que cada um deles pode apresentar uma atividade como dreno diferenciada, de acordo com o seu desenvolvimento. Os lóculos que apresentam maior força como dreno crescem mais, causando o entortamento do fruto.

Kanahama (1989) concluiu que o entortamento do fruto aumentava com temperaturas mais altas. Tiedjens (1928) relatou uma produção de maior número de frutos deformados em dias com intensidade luminosa reduzida.

A redução da área foliar de plantas de pepino provoca diminuição significativa no peso total da planta e peso de matéria fresca e seca dos frutos (Ramirez et al., 1988). Segundo Pharr, citado por Ramirez et al. (1988), a produção de fotossintetizados pelas folhas é fundamental para a produção de frutos de qualidade em pepino. Existe um equilíbrio entre crescimento vegetativo (hastes e folhas) e reprodutivo (frutos), caracterizado pela relação fonte: dreno (Marcelis, 1991).

O objetivo deste trabalho foi estudar a influência da redução de área foliar sobre a produção e qualidade dos frutos de pepino, assim como a importância da remoção dos frutos tortos na produtividade e qualidade dos remanescentes.

\section{MATERIAL E MÉTODOS}

O experimento foi conduzido sob ambiente protegido na Fazenda Experimental São Manuel, localizada no município de São Manuel - SP, pertencente à Faculdade de Ciências Agronômicas (FCA) da Universidade Estadual Paulista (UNESP), Campus de Botucatu.

Foram avaliados seis tratamentos:

1) Testemunha: planta com crescimento livre, sem desbaste de frutos, nem desbrotas;

2) Padrão: foram feitos todos os tratos culturais necessários como: eliminação dos brotos laterais até $04^{\circ}$ nó e as ramas laterais seguintes foram desbrotadas deixando somente 2 nós por rama. Realizou-se a poda da gema apical da haste principal quando esta atingia a altura do arame (cerca de 1,8 $\mathrm{m}$ );

3) Padrão com desbaste de frutos: foi feito semeIhante ao padrão, mas foram retirados todos os frutos que apresentaram entortamento quando ainda jovens $(<5 \mathrm{~cm})$;

4) Desfolhação I: tratos semelhantes ao padrão, mas com eliminação de $25 \%$ das folhas, mantendo-se três e retirando-se uma;

5) Desfolhação II: tratos semelhantes ao padrão, mas com eliminação de $50 \%$ das folhas em ordem alternada, isto é, folha sim, folha não;

6) Desfolhação III: tratos semelhantes ao padrão, mas com eliminação de $75 \%$ das folhas, mantendo-se uma e retirando-se três.
As retiradas das folhas foram realizadas a partir do $4^{\circ}$ nó, quando as folhas apresentavam-se totalmente expandidas. Assim, no tratamento 4, por exemplo, deixouse as folhas do $5^{\circ}, 6^{\circ}$ e $7^{\circ}$ nó e retirou-se a do $8^{\circ}$, deixouse as três seguintes e retirou-se a do $12^{\circ}$ e assim por diante. O mesmo foi feito nas ramas laterais. Após a primeira, as demais desfolhas, assim como as desbrotas e desbastes de frutos, foram realizadas uma ou duas vezes por semana, de acordo com a velocidade de crescimento das plantas.

O pepino híbrido Hokuho $\mathrm{n}^{\circ} 2$ foi enxertado (enxertia tipo fenda) sobre a abóbora 'Excite lkky' e as mudas foram transplantadas em 09/01/98, em um espaçamento de $1,0 \times 0,5 \mathrm{~m}$. O sistema de irrigação foi por gotejamento e a adubação constou de uma aplicação de composto por ocasião do preparo do solo $\left(5 \mathrm{~L} \mathrm{~m}^{-2}\right)$, adubo formulado 4-14-8 (150 $\mathrm{g} \mathrm{m}^{-1}$ de sulco) e nitrocálcio (20 $\mathrm{g} \mathrm{m}_{-1}$ de sulco). As adubações em cobertura foram realizadas a cada 14 dias com $5 \mathrm{~g}$ de nitrocálcio por planta.

Após o transplante, foram realizados os tratos culturais de acordo com a necessidade das plantas. As pulverizações foram realizadas em grande número (aproximadamente a cada 7 dias), para evitar perdas de área foliar por problemas fitossanitários. Apesar disto, no final do ciclo, com a suspensão das pulverizações no período das colheitas, as perdas de área foliar devido ao oídio (Erisiphe cichoracearum De Candolle) foram intensas, determinando o final da colheita. Algumas plantas tiveram que ser eliminadas devido a infecção por vírus (mosaico).

As colheitas foram realizadas três vezes por semana, sendo a primeira realizada no dia 02/02/98 e a última 03/04/98. Os frutos eram colhidos quando apresentavam um comprimento de aproximadamente $20 \mathrm{~cm}$.

Quando as primeiras plantas já atingiam a altura do arame para realização da desbrota apical, foi realizada uma avaliação de altura das plantas, e quando todas já haviam atingido este estádio, foram contados os números de nós por planta. Ao final do ciclo, foram contadas 0 número de ramificações laterais por planta e, assim, obtida a porcentagem de nós (a partir do $4^{\circ}$ ) com emissão de brotações laterais.

Em cada colheita contava-se o número de frutos totais e os que se apresentavam tortos ou com defeitos (daqui em diante denominados apenas como tortos) por parcela, assim como o peso dos mesmos. A partir destes dados, obteve-se a produção "comercial" (total-tortos) e a porcentagem de frutos tortos.

$O$ delineamento experimental utilizado foi em blocos ao acaso, com 5 repetições e 5 plantas por parcela. Após as análises de variância as médias dos tratamentos foram comparados pelo teste de Duncan ao nível de $5 \%$ de probabilidade.

Realizou-se, também, uma análise de regressão para os seguintes parâmetros: número de frutos total e comerciais e produção de frutos total e comerciais por planta, comparando os tratamentos padrão $(0 \%$ de desfolha), $25 \%, 50 \%$ e $75 \%$ de desfolha. 


\section{RESULTADOS E DISCUSSÃO}

Apesar de não ter havido diferença significativa entre os tratamentos para altura média das plantas (TABELA 1), observou-se que as plantas nos tratamentos onde foram realizados as desfolhas apresentaram maior número de nós, ou seja, os entrenós eram mais curtos e menos vigorosos. Segundo Aung \& Kelly (1966), com a desfolha em tomateiro, a planta reagia aumentando o crescimento das folhas remanescentes e reduzindo o da haste e raízes. Provavelmente foi o que ocorreu no presente trabalho, com redução no crescimento de cada entrenó individualmente.

Os tratamentos 1, 5 e 6 apresentaram menor porcentagem de nós com ramificações laterais comparativamente ao tratamento 3 , que foi onde se eliminou todos os frutos tortos $(<5 \mathrm{~cm})$. Provavelmente, este tratamento por não ter tido sua área foliar (fonte) afetada e com a eliminação de alguns drenos importantes (frutos novos tortos) pode ter favorecido a emissão de maior número de ramificações laterais (novos drenos). Esta maior emissão de ramificações laterais favorece o aumento do número de flores femininas, visto que nas plantas monóicas de pepino, as flores femininas ocorrem principalmente nos ramos laterais (Ramalho, 1973). Já no tratamento 1 as plantas cresciam livremente, sem desbrota ou desbate, o que deve ter proporcionado uma inibição da emissão de novas brotações (novos drenos), enquanto que com desfolha em excesso (tratamentos 5 e 6 ) as plantas apresentaram pequenas áreas fotossinteticamente ativas (fonte reduzida), desfavorecendo a emissão de novas brotações laterais (novos drenos). Estes resultados demonstram o balanço existente entre o número de drenos (flores, frutos e brotações novas) e de fontes (folhas fotossinteticamente ativas), conforme relatado por Marcelis (1991).

Quanto a porcentagem de frutos tortos (TABELA 1), o tratamento 3 foi o que apresentou menor valor diferindo de todos os demais, entretanto ainda apresentava $29 \%$ de frutos tortos. No início da colheita a incidência de doenças foliares era baixa e com isso a quantidade de frutos tortos foi pequena (menos de $10 \%$ nos primeiros 20 dias de colheita, no tratamento 3). Após esta época, houve um aumento da porcentagem de frutos tortos em todos os tratamentos devido a incidência de doenças e a redução de área foliar fotossinteticamente ativa. Também Demattê (1978) observou uma redução na proporção de frutos comerciais com o aumento da idade das plantas. Com isto, mesmo os frutos com mais de $5 \mathrm{~cm}$ de comprimento do tratamento 3 sofreram o entortamento devido a fatores fitossanitários e nutricionais.

Também observou-se que uma desfolha drástica (tratamento 6), favoreceu ainda mais o aumento da porcentagem de frutos tortos, mostrando a importância da área foliar não só na produção de frutos como também na qualidade dos mesmos, confirmando os relatos de Kanahama \& Saito (1984, 1985a).

Quanto a produção de frutos em peso e número por planta (TABELA 2), obtiveram-se os maiores valores no tratamento com plantas de crescimento livre, diferindo

TABELA 1 - Médias da altura das plantas, número médio de nós por planta, porcentagem de nós com ramificações laterais e de frutos tortos, com respectivas comparações pelo teste de Duncan (5\%). São Manuel - SP, 1998.

\begin{tabular}{ccccc}
\hline Tratamento & $\begin{array}{c}\text { Altura média das } \\
\text { plantas }(\mathrm{cm})\end{array}$ & $\begin{array}{c}N^{\circ} \text { médio de nós } \\
\text { por planta }\end{array}$ & $\begin{array}{c}\text { \% de nós com } \\
\text { ramos laterais }\end{array}$ & \% de frutos tortos \\
\hline 1 & $168,25 \mathrm{a}$ & $23,70 \mathrm{c}$ & $42,48 \mathrm{bc}$ & $34,76 \mathrm{~b}$ \\
2 & $169,80 \mathrm{a}$ & $23,92 \mathrm{c}$ & $49,46 \mathrm{abc}$ & $44,35 \mathrm{~b}$ \\
3 & $175,00 \mathrm{a}$ & $24,26 \mathrm{bc}$ & $65,20 \mathrm{a}$ & $29,02 \mathrm{c}$ \\
4 & $172,20 \mathrm{a}$ & $25,18 \mathrm{abc}$ & $57,82 \mathrm{ab}$ & $38,15 \mathrm{~b}$ \\
6 & $150,40 \mathrm{a}$ & $26,62 \mathrm{a}$ & $40,04 \mathrm{c}$ & $34,65 \mathrm{~b}$ \\
\hline
\end{tabular}

Médias, dentro de colunas, seguidas pela mesma letra não diferem estatisticamente pelo teste de Duncan a $5 \%$.

TABELA 2 - Produção (peso e número) de frutos total e comerciais por planta e peso médio dos frutos com respectivas comparações pelo teste de Duncan (5\%). São Manuel - SP, 1998.

\begin{tabular}{cccccc}
\hline Tratamento & $\begin{array}{c}\text { Número de } \\
\text { frutos total }\end{array}$ & $\begin{array}{c}\text { Número de frutos } \\
\text { comerciais }\end{array}$ & $\begin{array}{c}\text { Produção de } \\
\text { frutos }(\mathrm{g})\end{array}$ & $\begin{array}{c}\text { Produção de frutos } \\
\text { comerciais }(\mathrm{g})\end{array}$ & $\begin{array}{c}\text { Peso médio de } \\
\text { frutos }(\mathrm{g})\end{array}$ \\
\hline 1 & $31,96 \mathrm{a}$ & $18,45 \mathrm{ab}$ & $5691,3 \mathrm{a}$ & $3525,7 \mathrm{ab}$ & $192,7 \mathrm{a}$ \\
2 & $27,64 \mathrm{ab}$ & $16,01 \mathrm{~b}$ & $4893,4 \mathrm{ab}$ & $2843,0 \mathrm{~b}$ & $186,7 \mathrm{a}$ \\
3 & $29,33 \mathrm{ab}$ & $20,53 \mathrm{a}$ & $5428,7 \mathrm{ab}$ & $4268,9 \mathrm{a}$ & $195,0 \mathrm{a}$ \\
4 & $28,82 \mathrm{ab}$ & $15,58 \mathrm{~b}$ & $4922,7 \mathrm{ab}$ & $2929,8 \mathrm{~b}$ & $184,7 \mathrm{a}$ \\
5 & $20,37 \mathrm{bc}$ & $11,11 \mathrm{bc}$ & $3336,0 \mathrm{bc}$ & $2057,0 \mathrm{bc}$ & $186,4 \mathrm{a}$ \\
6 & $14,78 \mathrm{c}$ & $5,36 \mathrm{c}$ & $2244,0 \mathrm{c}$ & $931,3 \mathrm{c}$ & $174,7 \mathrm{a}$ \\
\hline
\end{tabular}

Médias, dentro de colunas, seguidas pela mesma letra não diferem estatisticamente pelo teste de Duncan a $5 \%$. 
significativamente dos tratamentos com 50 e $75 \%$ de desfolha. Entretanto, o tratamento 1 apresentou maior dificuldade de colheita devido a excessiva quantidade de massa verde. Além desta dificuldade, um grande número de frutos entortou (cerca de $35 \%$, TABELA 1), reduzindo a qualidade dos mesmos.

O tratamento padrão com eliminação dos frutos novos (tratamento 3 ) foi o que apresentou maior número e peso de frutos comerciais por planta (TABELA 2), diferindo significativamente de quase todos os tratamentos, com exceção do tratamento 1 . Conforme discutido anteriormente, no tratamento 3 , a eliminação dos frutos novos favoreceu a emissão de brotações laterais e de mais flores femininas. Assim as perdas com a eliminação dos frutos tortos foi compensada pelos novos frutos de melhor qualidade nestas brotações laterais, levando a um aumento no número de frutos comerciais em relação aos demais tratamentos. Comparando-se com o padrão (tratamento 2), este aumento foi de mais de 4 frutos comerciais por planta, ou seja, $25 \%$ de incremento.

Os resultados obtidos com $50 \%$ de desfolha são semelhantes aos obtidos por Parr \& Hussey, citados por Demattê (1978), que verificaram uma redução de $25 \%$ no número de frutos com a remoção de $50 \%$ de área foliar. Também Ramirez et al. (1988) observaram uma redução da produção de frutos de pepino, só que no estado maduro, com redução na área foliar da planta.

Apenas $25 \%$ de desfolha não afetou significativamente a produção e a qualidade dos frutos comparativamente ao tratamento padrão (TABELAS 1 e 2), talvez porque em pepino, a semelhança que ocorre em tomate (Tanaka \& Fujita, 1974 e Wolk et al., 1983), as folhas remanescentes sejam estimuladas a aumentar a taxa fotossintética e compensam uma pequena perda de área foliar.

Não se observou diferença para peso médio de frutos entre os tratamentos, ao contrário do observado por Ramirez et al. (1988), que relatou decréscimo no peso médio com a redução na área foliar. O motivo de tal diferença de resultados é apenas reflexo do estádio em que foram avaliados os frutos. Enquanto estes autores avaliaram frutos maduros, neste experimento os frutos eram colhidos no estádio imaturo, sempre com aproximadamente o mesmo tamanho (cerca de $20 \mathrm{~cm}$ de comprimento).

Para todas as características em que se fez análise de regressão para avaliar somente o efeito dos níveis de desfolha, a regressão linear foi significativa a 1\% de probabilidade e as equações obtidas foram as seguintes:

$$
\begin{aligned}
& \text { NTF }=29,9556-0,188016 . \text { desf } \quad\left(R^{2}=0,613^{\star \star}\right) \\
& \text { NFC }=17,479-0,14564 . \text { desf } \quad\left(R^{2}=0,721^{* \star}\right) \\
& \text { PTF }=5279,249-38,13928 . \text { desf } \quad\left(R^{2}=0,629^{\star *}\right) \\
& \text { PFC }=3181,4766-26,4323 . \text { desf } \quad\left(R^{2}=0,658^{* \star}\right)
\end{aligned}
$$

Onde:

NTF = Número total de frutos por planta;

$\mathrm{NFC}=$ Número de frutos comerciais por planta;

PTF = Produção $(\mathrm{g})$ total de frutos por planta;

PFC $=$ Produção $(\mathrm{g})$ de frutos comerciais por

planta;

desf $=$ representa porcentagem de desfolha.

$\mathrm{Na}$ Figura 1 estão representados os gráficos para as equações de NFT e NFC, enquanto que na Figura 2 para PTF e PFC. Em todas as características, observase uma redução na produção com o aumento do nível de desfolha das plantas.

Pelos resultados obtidos, com uma desfolha de $10 \%$ tem-se uma redução na produção total de 1,88 frutos/ planta, sendo que 1,46 desses frutos são comerciais, ou ainda, uma redução de $381 \mathrm{~g}$ por planta da produção total, sendo que $264 \mathrm{~g}$ seriam de frutos comerciais. Considerando o espaçamento utilizado $(1,0 \times 0,5 \mathrm{~m})$, teríamos 20.000 plantas ha ${ }^{-1}$ e uma redução de 29.200 frutos comerciais, ou ainda, $5,28 \mathrm{t} \mathrm{ha}^{-1}$.

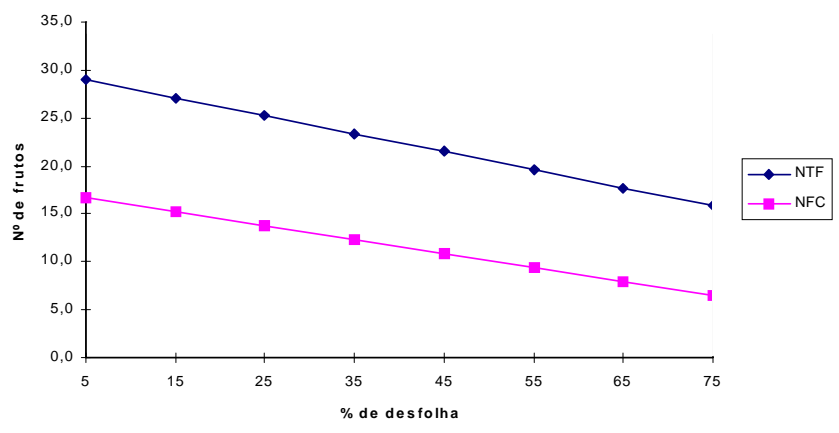

Figura 1 - Número de frutos total (NTF) e comerciais (NFC) por planta de acordo com a porcentagem de desfolha. São Manuel - SP, 1998.

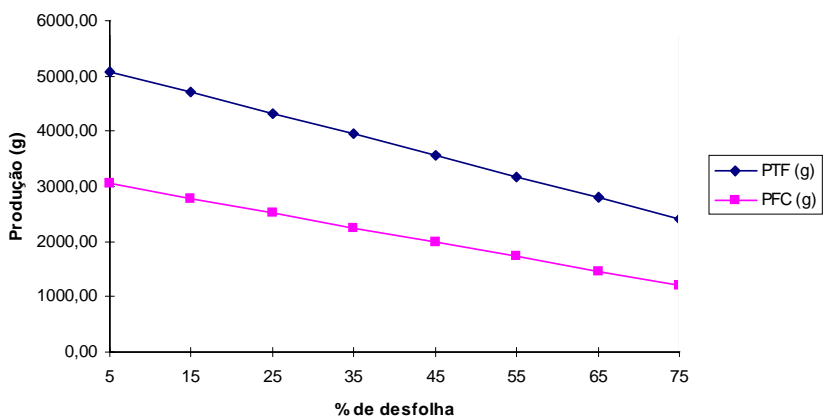

Figura 2 - Produção de frutos total (PTF) e comerciais (PFC) por planta de acordo com a porcentagem de desfolha. São Manuel - SP, 1998

\section{CONCLUSÃO}

Apesar da redução na produção e qualidade dos frutos de pepino ter sido proporcional ao nível de perda de área foliar, as plantas suportaram até $25 \%$ de desfolha sem decréscimo na produção. Além disso, a remoção de frutos novos tortos promoveu uma maior emissão de ramificações laterais e aumentou a produção de frutos comerciais. 


\section{AGRADECIMENTO}

Projeto financiado pela FAPESP, processos 97/

07577-7 (Bolsa de Iniciação Científica do primeiro autor) e 97/08175-0 (Auxílio à Pesquisa).

\section{REFERÊNCIAS BIBLIOGRÁFICAS}

AUNG, L.H.; KELLY, W.C. Influence of defoliation on vegetative, floral and fruit development in tomatoes (Lycopersicon esculentum Mill.). Journal of American Society for Horticultural Science, v.89, p.563-570, 1966.

DEMATTÊ, M.E.S.P. Comportamento de cultivares de pepino (Cucumis sativus L.) em duas épocas de semeadura. Piracicaba, 1978. 109p. Dissertação (Mestrado) - Escola Superior de Agricultura "Luiz de Queiroz", Universidade de São Paulo.

DENNA, D.W. Effects of genetic parthenocarpy and gynoecious flowering habit on fruit production and growth of cucumber Cucumis sativus L. Journal of American Society for Horticultural Science, v.98, p.602-604, 1973.

FNP Consultoria \& Comércio. Agrianual 2000. São Paulo, 2000. $546 p$.

KANAHAMA, K. Various factors related to curved in cucumber (1). Cultivation conditions and the occurrence of curved fruit. Agriculture and Horticulture, v.64, p.47-52, 1989.

KANAHAMA, K.; SAITO, T. Effects of planting and shading on fruit curvate in cucumber. Journal of the Japanese Society for Horticultural Science, v.53, p.331-337, 1984.

KANAHAMA, K.; SAITO, T. Effects of leaf number, fruit number and the shading of plants on fruit curvate in cucumber. Journal of the Japanese Society for Horticultural Science, v.54, p.216-221, 1985a.

KANAHAMA, K.; SAITO, T. Curvate and recovery from it in cucumber ovary and fruit. Journal of the Japanese Society for Horticultural Science, v.54, p.357-363, 1985b.

KANAHAMA, K.; SAITO, T. Carbohydrate distribution and ${ }^{14} \mathrm{C}$ photosynthates uptake in the curved fruits of cucumber. Journal of the Japanese Society for Horticultural Science, v.57, p.448-453, 1988.
LOWER, R.L.; EDWARDS, M.D. Cucumber breeding. In: BASSET, M.J. (Ed.) Breeding vegetable crops. Westport: Avi Publishing, 1986. cap.5, p.173-207.

MARCELIS, L.F.M. Effects of sink demand on photosynthesis in cucumber. Journal of Experimental Botany, v.42, p.1387-1392, 1991.

OLIVEIRA, A.C.B.; ARAÚJO, M.L.; LEAL, N.R. Avaliação do comportamento de três cultivares de pepino submetidas ao cultivo sob estrutura de proteção e a céu aberto na região Norte Fluminense - RJ. Horticultura Brasileira, v.13, p.99, 1995.

RAMALHO, M.A.P. Hábito de florescimento e frutificação do pepino (Cucumis sativus L.). Piracicaba, 1973. 48p. Dissertação (Mestrado) - Escola Superior de Agricultura "Luiz de Queiroz", Universidade de São Paulo.

RAMIREZ, D. R.; WEHNER, T.C.; MILLER, C.H. Source limitation by defoliation and its effect on dry matter production and yield of cucumber. HortScience, v.23, p.704-706, 1988.

REIS, N.V.B.; HORINO, Y.; OLIVEIRA, C.A.S.; BOITEUX, L.S. Influência da radiação fotossinteticamente ativa (RFA) sobre os componentes de produção de nove genótipos de pepino plantado a céu aberto e sob estufas plásticas. Horticultura Brasileira, v.1, p.55, 1991.

REIS, N.V.B.; HORINO, Y.; OLIVEIRA, C.A.S.; BOITEUX, L.S.; LOPES, J.F. Influência de temperatura - graus-dia sobre a produção de pepino sob cultivo protegido e a céu aberto. Horticultura Brasileira, v.10, p.65, 1992.

SILVA, A.A.; SOPRANO, E.; VIZZOTTO, M.S. Caracterização de deficiências nutricionais em pepineiro. Santa Catarina: EPAGRI, 1995. 35p.

TANAKA, A.; FUJITA, K. Nutrio-physiological studies on the tomato plant. IV. Source-sink relationship and structure of the source-sink unit. Soil Science and Plant Nutrition, v.20, p.305-315, 1974.

TIEDJENS, V.A. The relation of environment to shape of fruit in Cucumis sativus $\mathrm{L}$. and its bearing on the genetic plants. Journal of Agricultural Research, v.36, p.795-809, 1928.

WOLK, J.O.; KRETCHMAN, D.W.; ORTEGA JUNIOR., D.G. Response of tomato to defoliation. Journal of American Society for Horticultural Science, v.108, p.536-540, 1983.

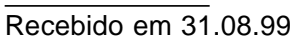

